\title{
Tombeau pour Léonore. Inceste et filiation chez Christine Angot
}

\author{
Évelyne Ledoux-Beaugrand
}

This text explores the issue of incest between mother and daughter from the perspective of filial desire in a number of texts by Christine Angot. While the incest between father and daughter is described as a loathsome crime, which left the narrator scarred, the incest between mother and daughter is considered by Angot as a sign of love, a sign of gratefulness. The question remains: how can incest lead to the establishment of a female genealogy? How can a mother's sexual desire for her daughter preserve the link between them? Tombeau pour Léonore explores these questions through psycho-analytical writings on incest and mother-daughter relationships.

« Obsessionnellement fixée sur l'inceste » (Angot 1999b, 29), comme elle se plaît à le répéter, Christine Angot l'est effectivement. Avant même la parution de L'inceste, sa relation incestueuse à son père hantait déjà la plupart de ses écrits. Le moins qu'on puisse dire, c'est que cet inceste, loin de taire son occurrence, Angot le reconnaît, retourne sans cesse vers lui, et le renouvelle dans chacun de ses récits. Or, la reconnaissance est justement ce qui fait défaut dans sa relation avec son père. Père qui, paradoxalement, l'inceste sans la reconnaître comme sa fille, sans reconnaître leur filiation qui fait de leur relation un amour incestueux. À cet inceste père-fille dans lequel les contacts sexuels ne s'accompagnent d'aucune reconnaissance filiale, Christine Angot oppose un inceste mère-fille fait, celui-là, d'amour. Différent de l'inceste père-fille qui expulse la narratrice hors de sa généalogique féminine, la laissant seule, héritière d'un patronyme qui ne l'inclut dans aucune lignée, le désir incestueux que manifeste explicitement Christine Angot pour sa fille est avant tout un désir de reconnaissance et de filiation.

Désir filial et inceste peuvent sembler incompatibles, mais il n'en est rien dans l'œuvre de Christine Angot. En raison de ce qu'elle nomme sa structure mentale incestueuse, Angot " associe ce qu'on n'associe pas, [...] recoupe ce qu'on ne recoupe pas. Chien-enfant, inceste-homosexualité ou sida, cousinecouple, blonde-conne, fric-haine, vedette-chienne, Léonore-or, charnier-mine d'or, holocauste-ghetto, ouvrier-noir, etc., etc. " (1999a, 105). À cette longue énumération de substantifs à première vue disparates, j'ajouterais le couple filiation-désir incestueux. Ainsi, le désir sexuel de la narratrice, dirigée vers sa fille, ne naît-il pas d'une volonté de reconnaissance, n'est-il pas une tentative de filiation ou une façon de recréer une généalogie qu'a rompue le père en transgressant l'interdit de l'inceste? Il semble, à la lecture des récits de Christine 
Angot, que cette dernière, notamment par son désir sexuel pour son enfant dans Léonore, toujours et par sa volonté de « marquer [...] chaque jour quelque chose sur elle, au moins une ligne » $(1997,11)$, cherche à inscrire sa fille dans une lignée féminine et, du coup, à redéfinir la relation mère-fille que la psychanalyse, dans le sillage des travaux de Freud sur la féminité, nous a appris à penser en termes de rupture et d'éloignement.

\section{Une liaison}

L'exil hors du féminin et la fin de la relation privilégiée entre mère et fille, voilà les deux conditions, selon Freud, du devenir-femme et surtout du devenir-femme « normale » de toute petite fille. Alors que Freud insiste sur « la nécessaire déliaison de ce lien si intense » (Kofman 195, je souligne), Christine Angot pense sa relation à Léonore en termes de liaison, à entendre ici au sens de liaison amoureuse, et même sexuelle. Dans Interview (1995), la narratrice déplore ce qu'elle considère comme le travail de la nature, c'est-à-dire la séparation de la mère et de la fille qui, auparavant, s'aimaient et formaient, à elles deux, « une âme dans une étoile » (21). Mais est-ce bien la nature qui veut cet éloignement, qui veut qu'après le « terremotto » (Angot 1995, 29), ce tremblement de terre de la naissance où mère et fille vont aux frontières du monde ensemble, une mère « ça ne sert peut-être jamais à rien » (58)? Luce Irigaray ne peut s'empêcher de voir des raisons culturelles à cette mise à distance des corps de la mère et de la fille: la culture occidentale interdirait non seulement, « le corps-àcorps avec la mère » $(1987,26)$, mais le désir même de la mère, son désir à elle pour ses enfants, ainsi que le désir qu'a sa fille pour elle. Si le tabou de l'inceste s'adresse autant au garçon qu'à la fille, il semble que la rupture entre le garçon et sa mère soit moins brutale. André Green remarque que « [l]e garçon lutte énormément avant de s'incliner devant la prohibition de l'inceste. Mais sa défaite est partielle parce qu'il a droit à un lot de consolation : "Pas ta mère, mais si tu en trouves une qui lui ressemble, cela ira, on fermera les yeux" » (39-40). Pour la fille, pas de lot de consolation puisque elle-même fait office d'objet de remplacement pour le garçon.

Cet interdit du corps-à-corps avec la mère, interdit qui touche avant tout la fille, aurait pour double conséquence l'impossibilité d'établir une généalogie féminine et la mise en place de ce que Luce Irigaray nomme « une hétérosexualité normative » (32). À cette hétérosexualité résultant de la séparation de la fille et de la mère, Irigaray oppose ce qu'elle appelle « entre beaucoup de guillemets: ““"homo-sexualité secondaire"”" » (32). Par ce terme, elle veut désigner un amour entre mère et fille ou, pour reprendre ses mots exacts, un « amour pour les femmes-sœurs » (32) différent de l'amour archaïque que les enfants des deux sexes portent à leur mère. Cette homosexualité secondaire entre mère et fille, Christine Angot, plus radicale que Luce Irigaray, la nomme inceste. Mais cet inceste dont parle Angot à propos de sa fille Léonore n'est en rien un passage à l'acte, et relève plutôt du désir : " Je ne veux pas faire d'inceste avec elle physiquement. Mais dans la tête, ce n'est pas possible autrement ") (1997, 12). Même si Léonore, qu'elle surnomme « [m]on amour comme une bite dressée » (15), 
elle « [s]e la ferai[t] bien dès maintenant » $(1997,22)$, Angot fixe néanmoins des limites à cet inceste. « Jamais ne rien embrasser d'ouvert sur elle » $(1995,125)$, décrète-t-elle dans Interview.

L'inceste avec Léonore est tout en surface et en retenue. « Heureusement que je n'ai pas de queue, ce serait terrible pour elle » $(1997,46)$, clame la narratrice de Léonore, toujours, faisant ainsi mine de braver l'interdit de l'inceste. Néanmoins, avant d'embrasser sa fille sur la bouche, de mettre enfin ses lèvres sur les lèvres de Léonore dont elle a tant envie, elle attend l'aval de Claude, son compagnon et père de Léonore. Cet inceste qu'elle désire au plus haut point mais auquel elle ne peut se résoudre tout à fait et pour lequel elle attend une autorisation qui ne vient pas, elle le vit donc par procuration, dans l'homosexualité. Monique Cournut-Janin, dans un article où elle questionne l'inceste mère-fille, s'intéresse brièvement à L'inceste de Christine Angot:

Serait-ce d'un retour à cette fusion incestueuse dont parlerait l'écrivain Christine Angot qui, dans son livre L'inceste, nous décrit d'abord les affres, les passions d'une relation fusionnelle homosexuelle, pour ensuite nous en donner l'avant-goût, à savoir l'inceste vécu, enfant, avec son père? (85)

S'il est vrai que l'« inceste paternel ne conduit certes pas nécessairement à l'homosexualité " (Cornut-Janin 85), la structure du récit d'Angot, dont la première partie est entièrement consacrée à sa relation fusionnelle, et pour le moins difficile, avec Marie-Christine alors que la seconde relate surtout l'inceste avec son père, laisse croire que son homosexualité, qu'elle dit par ailleurs être temporaire, découlerait de l'inceste père-fille. Ce retour au féminin à travers l'homosexualité, ce retour au « pays d'origine » (Angot 1999a, 53) devient, dès lors, une façon de réparer la lignée féminine qu'est venu rompre l'inceste commis par le père qui, comme n'a de cesse de le rappeler Angot dans plusieurs de ses récits, simultanément à ses actes incestueux, lui a légué son patronyme.

« [C]es femmes en contact » (1999a, 71) qui fascinent tant Angot se présentent à elle comme les maillons d'une chaîne qui avait été brisée, chaîne qui la lie non seulement à Marie-Christine, mais aussi à sa mère, et surtout à sa fille. Car plus qu'un retour au féminin, l'homosexualité, en raison de la duplication du même qu'elle met en jeu, est aussi considérée par Christine Angot comme une forme d'inceste': " Baiser avec une femme, tu as raison, c'est de l'inceste " (1999a, 36). Et j'ajouterais que baiser avec Marie-Christine, c'est connaître, indirectement certes, le plaisir charnel avec Léonore. Alors que la narratrice se plaît à rappeler qu'elle et Léonore ont le même corps, qu'elles sont « heureuse[s] d'être pareilles " $(1997,65)$ et même que sa fille est "l'intérieur de [s]on corps à la vue du monde » $(1995,124)$, l'identification de Léonore et de MarieChristine, voire la confusion entre elles est aussi très présente. Les relations sexuelles avec Marie-Christine ramènent sans cesse la narratrice vers sa fille: « Tu sais ma chérie? Quand elle remonte vers mon visage, le nom qui me vient sur les lèvres, c'est le tien ma belle. Lé-o-nore » $(1999 \mathrm{a}, 47)$. 
La confusion entre fille et amante est encore plus marquée dans ces longs passages de L'inceste où la narratrice répète, dans un long délire verbal, les noms Léonore et Marie-Christine, mêlant ceux-ci tout comme, dans certains passages, elle utilise le pronom « elle » sans que l'on sache s'il s'agit de sa fille ou de sa compagne:
j'accouchais Léonore Marie-Christine Marie-Christine Léonore Léonore Marie-Christine Marie-Christine Léonore Léonore Léonore Léonore Marie-Christine Léonore Léonore Léonore. [...] En accouchant je suis devenue homosexuelle en accouchant Léonore Marie-Christine Léonore Léonore. (1999a, 78-79)

Dans ces énumérations où prime Léonore, dont le prénom revient plus fréquemment, Marie-Christine, que la narratrice désigne souvent en utilisant seulement le prénom Marie, se pose comme le lien unissant Léonore à Christine. Elle est celle qui, littéralement, marie Léonore à Christine, marque l'union incestueuse de la mère et de la fille. Elle rend possible cet inceste si particulier car, comme l'affirme la narratrice, « l'inceste avec elle [Léonore], c'est seulement le bon côté » $(1997,85)$.

Lorsqu'elle parle des bons côtés de l'inceste, Angot fait référence à un désir incestueux, à un inceste imaginaire, tout en surface, sans pénétration, à un inceste vécu uniquement à travers l'écriture, bref à un amour mère-fille tout à fait différent de cet autre inceste qu'elle a connu avec son père. Car même si elle affirme, d'une part, comprendre le désir sexuel d'un parent pour son enfant et, de ce fait, comprendre mieux les agissements de son père avec elle, elle avoue, d'autre part, demeurer perplexe devant l'inceste paternel: « Du mal à des êtres qu'on aime tellement? » $(1997,22)$. En fait, l'inceste réel est distinct de l'inceste psychique; le second, si l'on en croit Jacques André, protégerait du premier. En des termes similaires, Nathalie Zaltman pose l'hypothèse suivante:

Je pense, mais ce n'est qu'une intuition, que, dans le passage à l'acte, il ne s'agit justement pas de la mise en acte d'un fantasme incestueux non refoulé ou mal refoulé. Il s'agirait plutôt d'une pratique sexuelle où les pulsions de destructions, les affects de haine inconsciente prédominent sur les pulsions érotiques. Le partenaire y est en place d'objet à consommer et à détruire davantage que d'objet amoureusement désiré. $(2001,62)$

Or chez Angot, bien que la narratrice craigne parfois de détruire, voire de tuer sa fille en l'étouffant sous cet immense désir qu'elle a pour elle, il s'agit bien d'amour, d'inceste psychique. Un inceste à un point tel psychique, en réalité, qu'il n'est pas tant dirigé vers Léonore que vers ce personnage fictif et de convention qu'est cette Léonore qui habite les pages de ses livres. Loin de la consommation et de la destruction, l'inceste mère-fille, chez Angot, se veut plutôt un acte de préservation. Il est l'écriture d'un amour mis à l'abri de la relation père-fille. 


\section{La marque}

Dans une entrevue accordée au magazine Têtu, Christine Angot explique être en état de guerre: « Mais je précise bien que c'est dans l'écriture. Moi je ne commets pas l'inceste, je ne suis pas un criminel, j'écris. La transgression est là: celle qui se situe dans la chair et celle qui se trouve sur le papier. ") (Le Dauphin 1999). Ainsi, Angot croit que " [1]a transgression suprême, c'est l'inceste, bien sûr » (Le Dauphin 1999), mais elle distingue l'inceste vécu dans la chair de l'inceste de papier, bref l'inceste réel, un crime qui laisse des blessures, et l'inceste psychique, voire, dans son cas, l'inceste textuel qu'elle commet uniquement par le biais du texte, de l'écriture. D'ailleurs, sa jouissance, elle ne la trouve pas dans ses contacts physiques avec Léonore, ni même dans les nombreuses scènes sexuelles qu'elles s'inventent et dans lesquelles sa fille tient toujours le rôle principal. C'est plutôt dans l'écriture, dans le fait de transposer sur la page ces scènes qui peuplent son imagination, que la narratrice ressent du plaisir sexuel:

Léonore trempe sa main dans la flaque d'eau et l'asperge. L'instant d'après, à quatre pattes, elle le supplie de la prendre à revers. Alors: à quatre pattes lui aussi. Il lui glisse la main en dessous, elle empoigne la queue et se la fourre dedans... jusqu'à la matrice, recta! Elle pousse encore un cri de cochon. Elle jouit, oh ça, elle jouit, elle jouira. Autant que moi quand je le marque. Quand je le vois dans ma tête, au contraire, non, c'est quand je le raconte. (1997, 32-33, je souligne)

Non seulement la narratrice marque-t-elle ces scènes sexuelles, jette-elle sur papier ses propres fantasmes qui mettent en jeu Léonore, mais c'est aussi, littéralement, sa fille qu'elle marque: «Je n'écris plus. Depuis aujourd'hui. Ça, ça ne s'appelle pas écrire, ça s'appelle marquer. Je marquerai chaque jour quelque chose sur elle, au moins une ligne » $(1997,11)$. Marquer équivaut, dans le langage familier, à écrire, inscrire et noter. Mais marquer, c'est aussi, au sens concret, « [d]istinguer, rendre reconnaissable (une personne, une chose parmi d'autres) au moyen d'une marque » (Le Grand Robert 2001, 1212). En somme, marquer constitue une forme de reconnaissance; la marque sert à établir une identité et, étant donné son caractère durable, à préserver cette identification. Or, dans l'œuvre de Christine Angot, s'opposent deux types de marques qui sont, l'une comme l'autre, liées à l'inceste. D'aucuns diront que l'inceste a marqué Christine Angot, voire qu'il a laissé sa marque à travers son œuvre. De fait, Angot affirme dans L'inceste: « Je suis marquée, la marque, et aussi le Mark, justement, la femme de mon père était allemande » (157). De même, elle soutient qu' « il n'y a qu'une chose qui compte, la marque. Et il m'a marquée » $(1999,208)$. Que ce soit la marque ou le Mark, le père est sans cesse associé à une forme de marquage, mais cette marque qu'il a laissée sur elle, cette marque incestueuse, ne s'accompagne d'aucune reconnaissance.

En raison de la « loi sur la filiation de 72 » (Angot 1999a, 180), le père d'Angot la reconnaît en ce sens qu'il lui transmet son nom et lui verse une pension 
jusqu'à sa majorité, mais cette reconnaissance n'est pas entière. Car en dehors des papiers officiels qui authentifient la filiation, personne ne sait que Pierre Angot est père d'une fille née avant son mariage avec Elizabeth. Lorsque qu'elle se rend à l'épicerie Codec et, avec l'autorisation de son père, veut faire porter ses achats sur le compte Angot, elle comprend que celui-ci a tu son existence à son entourage, et qu'en dehors des liens incestueux qui les unissent, elle n'est rien pour lui. Pas même une femme, car dans l'inceste qu'il commet sur elle, ils n'ont ensemble " que des relations sexuelles inabouties. Comme par hasard d'éphèbe... » $(1999 \mathrm{a}, 66)$. Ni fille, ni femme, elle n'est pour lui qu'un corps affalé sur le ventre dans lequel il trouve son plaisir. Mais ce corps sans visage et sans sexe, ce corps non identifié, Christine Angot, par l'écriture, le renverse:

C'est à ce moment-là que j'ai décidé de me retourner, de retourner mon corps, de me retourner moi. Pourquoi? Être considérée enfin comme une femme, pas comme un cul, un trou de cul, beurre sur la crêpe retournée, vaseline, je n'étais pas un cul, j'ai commencé de prendre le pouvoir à ce moment-là. [...] Proposer, me retourner de moi-même du bon côté, j'écrivais déjà, j'avais commencé. (1999a, 173)

De dessous, position qui était la sienne lors des relations anales avec son père, elle prend alors, avec sa venue à l'écriture, le dessus de l'histoire; c'est elle, désormais, qui guide le récit, c'est elle qui établit la filiation. Le lien incestueux l'unissant à son père, elle choisit de le rompre en écrivant: « Raser les murs, les barrières, au sens couper, rasoir, coupure de la veine et de la chance. Rasoir dans les murs de pierre prénom de mon père, sur cette pierre je bâtirai mon église, c'est la littérature, je l'entaille, un mur de livres, un mur de lamentations » (1999a, 3233 , je souligne).

En un sens, pour Angot, écrire - ou marquer - constitue une façon de rompre une lignée, en l'occurrence cette fausse lignée paternelle dans laquelle l'avait inscrite les gestes incestueux du père. Mais l'acte d'écriture s'avère aussi une entreprise de préservation: dans Léonore, toujours, la narratrice s'emploie à marquer chaque jour une ligne sur sa fille afin de garder intact ce qu'elle nomme " [c]et amour » $(1997,16)$, afin de préserver sa relation fusionnelle et incestueuse avec Léonore. Christine Angot cherche ainsi à contrer cet exil hors du féminin que, si l'on en croit Freud, devrait faire sien Léonore. Toutefois, la fusion, qui fait de la mère et de la fille une sorte d'entité bicéphale, ne peut perdurer indéfiniment. Cette « peau commune » (Anzieu 85), cette membrane symbolique rappelant l'enveloppe placentaire, qui lie Léonore à Christine, doit un jour disparaître. «[A]ssur[ant] entre les deux partenaires une communication sans intermédiaire, une empathie réciproque, une identification adhésive » (Anzieu 85 ), cette peau commune plonge la mère et la fille dans une « dépendance symbiotique mutuelle » (85) où toutes deux, à la longue, risquent d'étouffer. L'échec de sa disparition empêche, en effet, chacune d'elles d'acquérir sa propre peau, son propre Moi. Mais son effacement n'est pas synonyme pour autant de rupture complète: le lent détachement, jamais totalement complété, se fait plutôt 
sur le mode de l'oscillation, à l'image de Perséphone, figure de la mythologie grecque, qui effectue d'incessants allers-retours entre sa mère, Déméter, et son époux, Hadès ${ }^{2}$. Ainsi, si la proximité n'est plus celle que connaissaient mère et fille auparavant, si elles cessent de ne former qu'une seule personne, de partager le même corps, le lien qui les unit n'est pas rompu pour autant.

La relation mère-fille dans l'œuvre de Christine Angot est à l'image de ce mouvement oscillatoire. Consciente du danger qui guette sa fille si elle s'acharne à préserver cette peau commune, elle sait ne pas pouvoir demeurer indéfiniment dans cette proximité incestueuse avec Léonore. "Je suis folle d'elle et veut le rester $»^{3}(1997,15)$, affirme-t-elle tout en constatant que « Léonore s'éloigne quand même » (142). Dès lors, se pose pour elle la question suivante: comment préserver ce lien si intense sans tuer sa fille, sans laisser sur elle des marques permanentes, sans répéter avec elle l'inceste que son père lui a fait subir? Christine Angot en arrive à une solution pour le moins paradoxale: elle tue sa fille afin de préserver leur amour incestueux; elle met à mort Léonore afin d'assurer la survie de leur liaison. La fin abrupte et précipitée de Léonore, qui meurt des suites d'une chute, sous le regard attendri de ses parents, n'est pas sans surprendre. Pourtant, la mort de l'enfant évite la séparation que redoute tant la mère. Jacques André croit en effet que « [1]'enfant mort pourrait bien être l'enfant incestueux par excellence, celui dont jamais on ne se séparera, jamais plus. [...] Non seulement il n'est pas "mort", [...] mais il n'est pas tuable » $(2001,28)$. Ainsi, Léonore, toujours se présente comme le tombeau de Léonore : lieu de son décès - décès textuel, je le rappelle, car Léonore, l'enfant de Christine Angot, est bien vivante - mais aussi de sa survie, ou plutôt, lieu de la survie du lien incestueux qui unit la mère à la fille. Car si Léonore grandit, acquiert son autonomie, si s'effrite peu à peu cette peau commune à la mère et la fille, celles-ci demeurent indéfiniment liées, leurs deux noms accolés sur la couverture du livre, pour toujours.

\section{Conclusion}

L'œuvre de Christine Angot n'en est ni à une contradiction ni à un paradoxe près. "On peut utiliser toute la vie dans l'écriture heureusement", affirme-t-elle dans L'usage de la vie (17). « Tout, on peut tout dire, tout. Dans l'écrit. Que son enfant, on voudrait la voir morte " (17). Non seulement peut-on tout dire dans l'écrit, peut-on dire ce qui, généralement, se doit d'être tu, mais on peut même tuer son enfant, lui donner la mort. Une mort qui, paradoxalement, se veut comme un embaumement, voire une momification. Car cet infanticide littéraire garde intacte celle-là même qui est tuée ou, plutôt, garde intacte cette entité quasi indissociable que forment alors la mère et la fille. Ainsi, chez Angot, la mort peut devenir gage de survie, ou plutôt gage de la survie du lien mère-fille qui, nécessairement, s'assouplira lorsque Léonore acquerra son autonomie, de même que l'inceste peut se présenter à la fois comme un crime odieux qui laisse marques et blessures sur celle qui l'a subi et comme la preuve d'un immense amour, la marque de la reconnaissance que porte la mère pour sa fille. $\mathrm{Si}$, à l'interviewer qui lui demande, à propos de la relation mère-fille, « en quoi a-t-elle plus d'importance? La mise 
au monde? Le même sexe? $»(1995,20)$, Angot n'offre aucune réponse directe, elle souligne toutefois, à travers plusieurs de ses récits, l'importance de ce lien complexe qui, là où on le croit délié et dénoué, s'avère plutôt inextricable.

\section{Notes}

${ }^{i}$ Le retour au même est accentué dans le récit d'Angot par la similitude des prénoms de la narratrice et de sa compagne: Christine et Marie-Christine. Les nombreuses références religieuses qui traversent l'œuvre d'Angot - qui s'identifie à Sainte Christine martyrisée par son père, au Christ ou encore qui associe Léonore à un Christ qu'elle porte à bout de bras - laissent croire que Marie-Christine constitue une sorte de double immaculé de Christine. En effet, la présence du prénom Marie, référant à la vierge, fait d'elle un double qui n'a pas connu la pénétration, bref qui n'a pas connu l'inceste. Dans cette relation en miroir qui unit Christine et Marie-Christine, il y a, pour la narratrice, un retour à un temps d'avant l'inceste, un temps où elle était encore fortement liée à sa mère, voire un retour au « terremoto » (Angot 1995, 29) où mère et fille vont « à la frontière du monde ensemble $»(30)$.

ii Selon Marianne Hirsch (1989), le mythe de Déméter et Perséphone est plus approprié lorsque vient le temps de penser la relation mère-fille. Bouleversée par la disparition de Perséphone, qui a été enlevée par Hadès, Déméter, déesse du Blé, ne peut se résoudre à ne plus jamais revoir sa fille. Elle quitte alors l'Olympe à la recherche de cette dernière et avec son départ cessent les récoltes. Vu la menace de famine qui pèse sur les résidants de l'Olympe, Zeus intervient et trouve un compromis: Perséphone passera six mois de l'année avec sa mère et les six autres avec son époux.

iii La narratrice de Léonore, toujours (1997) peut être qualifiée, en reprenant les termes de Jacques André, de « mère folle ». La mère folle est celle qui refuse que «Un cesse, se divise, que deux advienne » $(2001,25)$. D'ailleurs, dans plusieurs passages, la narratrice affirme ne faire qu'une avec sa fille. Elle perçoit, par exemple, sa mort éventuelle comme « la séparation de [s]on corps » $(1997,39)$ puisqu'une partie d'elle-même, c'est-à-dire Léonore, demeurera vivante. De même, Angot écrit, dans L'inceste: "Mon enfant autrement dit ma chair autrement dit mon corps, ce que je suis, ma vie » $(1999 \mathrm{a}, 166)$.

\section{Bibliographie}

André, Jacques (dir.). Incestes. Paris: PUF, 2001.

— «e lit de Jocaste ». In Incestes. Paris: PUF, 2001. 9-28.

Angot, Christine. L'inceste. Paris: Stock, 1999a.

_ L'usage de la vie. Paris: Milles et une Nuits, 1999b.

Léonore, toujours. Paris: Fayard, coll. « Pocket », 1997.

Interview. Paris: Fayard, coll. « Pocket », 1995. 
Anzieu, Didier. Le Moi-peau. Paris: Dunod, 1995.

Cournut-Janin, Monique. « Incestes. Histoires et préhistoires 》. In Incestes. Jacques André (dir.). Paris: PUF, 2001. 75-90.

Freud, Sigmund. "La féminité ». Nouvelles conférences d'introduction à la psychanalyse. Paris: Gallimard, 1984. 150-181.

Green, André. 2001. " La relation mère-enfant nécessairement incestueuse ». In Incestes. Jacques André (dir.). Paris: PUF, 2001. 25-56.

Hirsch, Marianne. The Mother / Daughter Plot : Narrative, Psychoanalysis, Feminism. Bloomington et Indianapolis: Indiana University Press, 1989.

Irigaray, Luce. « Le corps-à-corps avec la mère ». Sexes et parentés. Paris:

Minuit, 1987. 21-33.

Kofman, Sarah. L'énigme de la femme. La femme dans les textes de Freud. Paris: Galilée, 1980.

Le Dauphin, Axelle. «Christine Angot ». Têtu, 39 (1999) http://tetu.com/ archives/1999-10/1/

Zaltzman, Nathalie. 2001. "L'inceste est-il une notion psychanalytique ». In Incestes. Jacques André (dir.). Paris: PUF, 2001. 57-74. 